\title{
A multicentre evaluation exploring the impact of an integrated health and social care intervention for the caregivers of ICU survivors
}

Joanne McPeake ( $\square$ joanne.mcpeake@glasgow.ac.uk)

NHS Greater Glasgow and Clyde

Philip Henderson

School of Medicine, Dentistry and Nursing, University of Glasgow

Pamela MacTavish

NHS Greater Glasgow and Clyde

Helen Devine

NHS Greater Glasgow and Clyde

Malcolm Daniel

NHS Greater Glasgow and Clyde

Phil Lucie

NHS Lanarkshire

Lynn Bollan

NHS Lanarkshire

Lucy Hogg

NHS Fife

Mike MacMahon

NHS Fife

Sharon Mulhern

NHS Ayrshire and Arran

Pauline Murray

NHS Ayrshire and Arran

Laura o'neill

NHS Lanarkshire

Laura strachan

NHS Lanarkshire

Theodore Iwashyna

University of Michigan-Ann Arbor

Martin Shaw

NHS Greater Glasgow and Clyde

Tara Quasim 
School of Medicine, Dentistry and Nursing, University of Glasgow

\section{Research Article}

Keywords: critical care, rehabilitation, family, caregivers and mental health

Posted Date: February 15th, 2022

DOI: https://doi.org/10.21203/rs.3.rs-1342666/v1

License: (c) (i) This work is licensed under a Creative Commons Attribution 4.0 International License. Read Full License 


\section{Abstract}

Background: Caregivers and family members of Intensive Care Unit (ICU) survivors can face emotional problems following patient discharge from hospital. We aimed to evaluate the impact of a multi-centre integrated health and social care intervention, on caregiver and family member outcomes.

Methods: This study evaluated the impact of the Intensive Care Syndrome: Promoting Independence and Return to Employment (InS:PIRE) programme across 9 sites in Scotland. InS:PIRE is an integrated health and social care intervention. We compared caregivers who attended this programme with a contemporary control group of ICU caregivers (usual care cohort), who did not attend.

Results: The primary outcome was anxiety measured via the Hospital Anxiety and Depression Scale at 12 months post hospital discharge. Secondary outcome measures included, depression, carer strain and clinical insomnia. A total of 170 caregivers had data available at 12 months for inclusion in this study. 81 caregivers attended the InS:PIRE intervention and completed outcome measures at 12 months posthospital discharge. In the usual care cohort of caregivers, 89 completed measures. The two cohorts had similar baseline demographics. After adjustment, those caregivers who attended InS:PIRE demonstrated a significant improvement in symptoms of anxiety (OR: $0.42,95 \% \mathrm{Cl}: 0.20-0.89, p=0.02$ ), carer strain (OR: 0.39; 95\% Cl:0.16-0.98 $p=0.04$ ) and clinical insomnia (OR: 0.40; 95\% Cl:0.17-0.77 $p<0.001$ ). There was no significant difference in symptoms of depression at 12 months.

Conclusions: This multicentre evaluation has shown that caregivers who attended an integrated health and social care intervention, reported improved emotional health and less symptoms of insomnia, 12 months after the delivery of the intervention.

\section{Background}

The profile of Intensive Care Unit (ICU) survivorship has been brought into sharp focus due to the COVID19 pandemic [1, 2]. Following a critical illness, patients can experience clinically important physical, emotional and cognitive symptoms [3-5]. These problems can have major implications for the patient and healthcare system $[6,7]$.

Less focus has been placed on family members and caregivers of ICU survivors. This vulnerable group often have a challenging trajectory during the critical care recovery period and are known to experience emotional and social problems [8-10]. However, to date, there are few interventions designed to address these issues, and none, to our knowledge, which have been successful at improving long-term outcomes in this group $[11,12]$.

Therefore, we aimed to evaluate the impact of a multi-centre integrated health and social care intervention on family members and caregivers outcomes. Specifically, we sought to understand what impact this intervention had on emotional outcomes and social disruption for the caregiver. 


\section{Methods}

This study evaluated the impact of an integrated health and social care intervention aimed at improving outcomes for ICU survivors and their caregivers in the year following hospital discharge. We compared caregivers who attended this intervention with a contemporary, usual care cohort (caregivers who had been exposed to critical care and were currently caring for a critical care survivor) who did not attend the programme. A caregiver was defined as the individual who provided most of the financial, emotional, and physical support for the patient, or the individual primarily responsible for caring for the patient on an unpaid basis [13]. Previous work has described the outcomes of patients who participated in this intervention, alongside a detailed process evaluation [14].

The study involved the expansion of the Intensive care Syndrome: Promoting Independence and Return to Employment (InS:PIRE) programme from a single general Intensive Care Unit (ICU), to four further ICUs throughout Scotland between 2016 and 2020. We recruited a contemporary usual care cohort from sites who had no active follow-up or rehabilitation for ICU survivors.

Using a multicentre cohort design we compared caregivers who attended the InS:PIRE intervention with a contemporary usual care cohort from sites who had no active follow-up for ICU survivors or caregivers. We report a cohort study, as per the Strengthening the Reporting of Observational Studies in Epidemiology (STROBE) guidelines [15].

\section{Approval}

Ethical approval was granted by the Northwest (Liverpool Central) Research Ethics Committee (reference number: 17/NM/0199). All participants provided written consent.

\section{Intervention}

The InS:PIRE programme is a complex intervention and has been described previously [16-17]. Briefly, all patients receive individual reviews with 1) an ICU doctor and nurse; 2) pharmacist; and 3) physiotherapist. These reviews offer a debrief of the ICU stay, an assessment of ongoing problems, goal setting, and patient directed management plans. Clinical Psychology services, alongside occupational therapy is available. Peer support was embedded in the programme using shared waiting areas, group sessions, and the presence of patient and caregiver volunteers who were further along the recovery trajectory [18]. Of note several group sessions were set up for caregivers exclusively, including the clinical psychological support. Every site involved local community organisations who provided welfare advice including information on relevant government benefits and housing. This support was delivered through a combination of individual appointments, drop-in sessions, or group discussions. Support for caregivers from local agencies was also integrated at every site. In contrast to other ICU recovery services which often include a single clinical appointment, InS:PIRE was designed as a recovery programme; patients and caregivers attend each week for five weeks and are followed up for one year after initial attendance. 
InS:PIRE was co-designed with local patients and caregivers. During this process, patients and caregivers both described the necessity for a specific and deliberate focus on caregivers within any rehabilitation service. This is also consistent with previous evidence is this field [19]. Initial single centre work showed that supporting caregivers was feasible, safe and acceptable, thus, this support was included in this multi-centre scale up $[20,21]$. Caregivers could attend all sessions and were eligible to receive the same support as patients. This only differed with the physiotherapy and pharmacy sessions which were aimed specifically at patient recovery.

Participants were invited to the programme between 4 and 12 weeks after hospital discharge. Patient inclusion criteria were any patient receiving level three care (multiple organ support and / or invasive respiratory support) or more than seven days of level two care (single organ support or postoperative care). There was no upper age limit for inclusion. Exclusion criteria were any patient who was terminally ill, had suffered a traumatic brain injury, or was an in-patient under psychiatric services. Caregiver inclusion criteria were: paired consented patient data had to be available and caregiving responsibilities occurred in an informal, non-paid basis. We limited inclusion in the research to one caregiver per patient, however, multiple caregivers could attend InS:PIRE.

\section{Intervention cohort}

Five sites piloted the InS:PIRE programme as part of a quality improvement collaborative (intervention cohort) over two years. Caregivers in the intervention cohort were consecutively recruited to this study between May 2016 to October 2018 (follow-up completed December 2019). Caregiver outcome measures were completed via a pre-planned 12-month follow-up appointment. Participants were given the opportunity to complete questionnaires at InS:PIRE attendance or via telephone.

\section{Usual care cohort}

Patients in the usual care cohort were recruited from eight hospitals in Scotland between 10-16 months post hospital discharge. Patients were identified by searching the local electronic records system and were recruited by postal survey. Questionnaire packs were sent to eligible patients with pre-paid return envelopes. Alongside these patient packs, we asked the patient's primary caregiver (or closest family member) to complete a separate pack. Reminder questionnaire packs were sent if the pack was not returned after one month. Participants were also given opportunity to call to discuss issues or recruitment with researchers. The usual care cohort were recruited between July 2017 and March 2020. Ethical approval was in place to continue beyond March 2020, however, the impact of the coronavirus disease 2019 pandemic was unknown, thus recruitment to this study was closed in an attempt to reduce any confounding effect.

\section{Data Collection}

We created a short questionnaire in order to collect family member demographics. Data collected included age, relationship to patient, and gender. 
In-hospital patient demographic and clinical data was obtained from clinical notes and discharge summaries. Patient comorbidity data (including mental health data) were obtained from medical notes and critical care admission records. Critical care length of stay was taken for the highest level of care and during the first critical care admission only.

The Scottish Index of Multiple Deprivation (SIMD) is produced by the Scottish Government as a measure of deprivation, with postcode areas defining data on socio-economic status. This research split the SIMD into five categories to define socio-economic status; quintile one represented the most deprived and quintile five the least [22].

\section{Outcome measures}

\section{Hospital Anxiety and Depression Scale}

The primary outcome measure in this study was caregiver anxiety. Anxiety and depression were measured using the Hospital Anxiety and Depression Score (HADS) [23]. The HADS questionnaire contains 14 statements relating to mood, with seven statements relating to depression and seven to anxiety. Each statement has four potential options (scored 0-3). The cut-off points widely adopted for HADS are described in S1. In this analysis we classified anxiety and depression as a score of 8 or above utilising the score subscales [23]. Appropriate licensing requirements were in place for the use of the HADS.

\section{Carer Strain Index}

Alongside the HADS, the Carer Strain Index (CSI) was utilised [24]. The CSI measures strain, including social strain, related to care provision from the caregiver's perspective. There are elements related to emotional adjustment, social issues, physical and financial strain. Each question is given one point; a score of seven or greater is the generally accepted cut off point for a high level of strain.

\section{The Insomnia Severity Index}

The Insomnia Severity Index (ISI) is a seven-item questionnaire which has been validated as a screening tool for clinical insomnia. Participants are asked to rank the severity of their sleep problems on a scale of zero to four and answer four other questions regarding satisfaction with their sleeping patterns [25]. We defined insomnia as a score of 8 and greater from a maximum score of 28 (range 0 to 28).

\section{Statistical Analysis}

Analyses was undertaken using R (Version 4.0.3). All missing covariates were imputed using categorical and regression trees analysis with the Multivariate Imputation by Chained Equations (MICE) software package. We used 5 imputations and 10 iterations. Information on missing data is presented in $\mathbf{S} 2$.

Continuous variables were expressed as medians and interquartile range (IQR). The Kruskall-Wallis test was used to compare different sub-groups and the chi-squared test to analyse categorical variables. 
Logistic regression was used to understand the impact of the intervention on the main outcomes of this study.

Models were created using domain knowledge and previous evidence in the field. All models were adjusted for: relationship with the patient; caregiver age; caregiver gender; time to follow up and socioeconomic status (SIMD quintiles). We also adjusted patient level demographics which are known to influence recovery including: hospital length of stay; patient age and the presence of a mental health comorbidity (pre-existing).

\section{Results}

\section{Demographics}

Two hundred and six patients attended InS:PIRE across the five participating sites and consented to participation in the study. There were 136 caregivers who attended InS:PIRE and consented to participation in this study. Eighty-one (60\%) of these caregivers completed outcomes measures at one year (intervention cohort).

In the usual care cohort, 452 patients were sent questionnaire packs across the four usual care cohort sites; 115 patients returned the packs for analysis in the study. Of these, $89(77 \%)$ caregivers completed outcome measures at one year and were included in the caregiver usual care cohort. The dataset was $96.2 \%$ complete; a breakdown of the missing variables is shown in $\mathbf{S}$.

The caregiver intervention and usual care cohort were similar in all baseline demographics. Both cohorts were predominantly female (intervention: 49 (60.5\%) vs usual care: 57 (64\%)) and had similar age profiles (intervention:58 (IQR: 48.0-66.3) vs usual care 58 (IQR: 47.7-69.0) years (Table 1). There was also a similar spread across the socio-economic gradient in both groups. Patient and caregiver demographics are shown in Table 1.

\section{Outcomes}

\section{Anxiety and Depression}

After adjustment, there was a $58 \%$ adjusted odds reduction of screening for anxiety (measured as a score of 8 or greater on the HADS anxiety scale) in those caregivers who received the InS:PIRE intervention (OR: $0.42,95 \% \mathrm{Cl}: 0.20-0.89, p=0.02$ ) (Table 2). The number of caregivers who had depression (classified as a score of 8 or greater on the HADS depression scale) was $27 \%$ in the usual care cohort vs $22 \%$ in the intervention cohort, however, the odds of screening for depression at 12 months was not significantly different in those who received the InS:PIRE intervention, (OR: 0.58, 95\% Cl:0.26-1.31, $p=0.19$ ) (Table 2).

\section{Carer Strain}


After adjustment, there was a significant, $61 \%$ reduction in the odds of carer strain in those who received the InS:PIRE intervention (OR: 0.39; $95 \% \mathrm{Cl}: 0.16-0.98 p=0.04$ ). The rate of strain (defined as a score equal to or greater than 7) was $30 \%$ in the usual care cohort compared to a rate of $19 \%$ in the intervention group. There were no significant differences between individual components of the CSI. Considering both groups together, $25 \%$ of caregivers indicated that there had been work adjustments during the recovery period and over one quarter $(26 \%)$ described financial strain.

\section{Insomnia}

After adjustment, there was a significant reduction in the odds of clinically important Insomnia, measured via the ISI in those caregivers who received the InS:PIRE intervention by $65 \%$ (OR: $0.36 ; 95 \%$ Cl:0.17-0.77 $p<0.001$ ) (Table 2). The incidence of insomnia (defined as an ISI score above 7 ) was $61 \%$ in the usual care cohort, and $38 \%$ in the intervention group.

A visual representation of the estimated impact of the InS:PIRE programme on caregiver outcomes at 12 months is shown in Figure 1. A full description of all outcome model estimates is provided in S3.

\section{Discussion}

This evaluation of a multicentre integrated health and social care intervention demonstrated a positive impact on the emotional health of ICU survivor caregivers at 12 months. As far as we can establish, this is the first multicentre intervention internationally to report benefit for caregivers during the post- ICU recovery phase.

The InS:PIRE intervention was designed as a peer supported, multi-disciplinary programme for both patients and caregivers [16]. Previous research has described multiple benefits of peer support for both patients and caregivers in the post-ICU setting such as reduced anxiety, improved external validation of progress, and support with expectation management [26, 27]. A further mechanism which we hypothesize has driven improvements, is the intentional focus on caregiver outcomes, rather than caregiver outcomes being viewed as a biproduct of patient treatment. Intentional use of separate caregiver education sessions alongside the integration of local community services which focused on caregiver needs, were available at every site. However, it is important to recognise that targeted treatments for both patients and caregivers are likely to have had a bidirectional effect across the family unit. That is, a patient knowing a caregiver is receiving the support they require will potentially have a positive effect on their recovery, and likewise a caregiver seeing the patient receiving the necessary support to recover will likely reduce anxiety and mental health problems.

Carer strain also significantly reduced for those caregivers who attended the programme. The Carer Strain Index is a tool which examines all the global aspects of caring responsibilities (S1). This includes domains such as return to employment, physical and financial strain [24]. Caregivers had access to social, financial and welfare advice for the duration of the programme; this may well have been the driver for the reduction in carer strain seen. This finding is consistent with work from primary care in the UK; 
improving the social and financial situation of people, by co-locating social and welfare services in health centres resulted in improvements in mental health [28]. Moreover, recent evidence has demonstrated a direct correlation between emotional and social outcomes in ICU survivors [29]. Future interventions aimed at addressing outcomes in this group must ensure that all aspects of an individual's wellbeing are considered.

Although there were less symptoms of depression in the InS:PIRE cohort, the intervention did not significantly improve symptoms of depression for caregivers at 12 months. Previous literature has demonstrated that symptoms such as depression improve over the first year of recovery for most critical care survivors [30]. We hypothesise that caregivers have a different recovery trajectory to patients, and thus, may require targeted interventions at different time periods. There is limited evidence describing the trajectories of symptoms beyond 2 months for caregivers following exposure to critical care illness [31]. Given the large volume of informal, unfunded care provided by this caregiver group in the months following hospital discharge, it is crucial that health services offer adequate support to this cohort. More research is urgently required in this area, in order to fully understand how best to support this vulnerable group, in relation to symptoms of depression.

\section{Limitations}

Study limitations are notable. Firstly, we do not know if the caregivers included in this study had preexisting mental health problems. As such we are unable to account for an imporntant baseline characteristic in our analysis. Secondly, this was not a randomised control trial. While there was substantial overlap in baseline characteristics between the intervention and usual care cohorts, they were not randomly assigned to either usual care or the intervention, as such, this limits the ability to draw casual inference from the results. Moreover, we experienced attrition over the course of the 12 months, common with other studies in the field, which may have also influenced the reported outcomes [11].

It could be argued that the patients and caregivers who attended the InS:PIRE programme, were more engaged and motivated about improving their health, thus, accounting for the improved outcomes seen in the intervention cohort. However, there was diversity in the cohort across the socio-economic gradient. Moreover, $40 \%$ of patients in the intervention cohort had multi-comorbidities and over a quarter had preexisting mental health issues. This would suggest that the intervention arm did include patients with significant chronic issues, who often have challenging trajectories following critical illness [32]. Finally, we only have data at one time point for the control cohort. As such we cannot fully understand the symptom trajectory for caregivers, which limits the interpretability of some of the results.

\section{Conclusions}

This multicentre evaluation has shown that caregivers who attended the InS:PIRE programme, reported improved emotional health and less symptoms of insomnia 12 months after hospital discharge, in comparison to a contemporary control group. More work is required to understand how the recovery 
trajectory for this group interacts with the patient recovery trajectory, to ensure services fully support all needs in this group.

\section{Abbreviations}

Acute Physiology And Chronic Health Evaluation: APACHE

Confidence Interval: $\mathrm{Cl}$

Carer Strain Index: CSI

Hospital Anxiety and Depression Scale: HADS

Intensive Care Unit: ICU

Intensive Care Syndrome: Promoting Independence and Return to Employment: InS:PIRE

Insomnia Severity Index: ISI

Interquartile Range: IQR

Length of Stay: LOS

Multiple Imputation by Chained Equations: MICE

Odds Ratio: OR

Scottish Index of Multiple Deprivation: SIMD

Strengthening the Reporting of Observational Studies in Epidemiology: STROBE

\section{Declarations}

Ethics Approval and consent to participate. All participants provided written informed consent. Ethical approval was granted by the Northwest (Liverpool Central) Research Ethics Committee (reference number: 17/NM/0199).

Consent for publication: Not applicable

Availability of data and materials: The datasets used and/or analysed during the current study are available from the corresponding author on reasonable request

Competing interests: The authors declare that they have no competing interests

Funding: The project was funded by an award from the Health Foundation (173544) and a THIS. Institute (University of Cambridge) Fellowship 307748-01/ PD-2019-02-16. 
Authorship contribution: JM and TQ: conceptualisation of the study

$\mathrm{JM}, \mathrm{PH}$ and $\mathrm{MS}$ conducted the analysis (formal analysis)

$\mathrm{JM}, \mathrm{MS}, \mathrm{PH}, \mathrm{TJ}$ contributed to the interpretation of the findings (validation).

All authors critically revised the paper for intellectual content and approved the final version of the manuscript.

Acknowledgements: The authorship team would like to thank the patients who took the time to participate in this study.

\section{References}

1. Hosey MM, Needham DM: Survivorship after COVID-19 ICU stay. Nature Reviews Disease Primers, 6(1):60.

2. Lone NI, McPeake J, Stewart NI, Blayney MC, Seem RC, Donaldson L, Glass E, Haddow C, Hall R, Martin C: Influence of socioeconomic deprivation on interventions and outcomes for patients admitted with COVID-19 to critical care units in Scotland: A national cohort study. The Lancet Regional Health-Europe 2021, 1:100005.

3. Iwashyna TJ, Ely EW, Smith DM, Langa KM, Iwashyna TJ, Ely EW, Smith DM, Langa KM: Long-term cognitive impairment and functional disability among survivors of severe sepsis. JAMA: Journal of the American Medical Association 2010, 304(16):1787-1794.

4. Wade DM, Howell DC, Weinman JA, Hardy RJ, Mythen MG, Brewin CR, Borja-Boluda S, Matejowsky $\mathrm{CF}$, Raine RA: Investigating risk factors for psychological morbidity three months after intensive care: a prospective cohort study. Critical Care 2012, 16(5):R192-R192.

5. Haines KJ, Hibbert E, McPeake J, Anderson BJ, Bienvenu OJ, Andrews A, Brummel NE, Ferrante LE, Hopkins RO, Hough CL: Prediction models for physical, cognitive, and mental health impairments after critical illness: A systematic review and critical appraisal. Critical care medicine 2020, 48(12):1871.

6. Cheung AM, Tansey CM, Tomlinson G, Diaz-Granados N, Matté A, Barr A, Mehta S, Mazer CD, Guest $\mathrm{CB}$, Stewart TE: Two-year outcomes, health care use, and costs of survivors of acute respiratory distress syndrome. American journal of respiratory and critical care medicine 2006, 174(5):538-544.

7. McPeake J, Mikkelsen ME, Quasim T, Hibbert E, Cannon P, Shaw M, Ankori J, Iwashyna TJ, Haines KJ: Return to Employment Following Critical Illness and Its Association with Psychosocial Outcomes: A Systematic Review and Meta-Analysis. Annals of the American Thoracic Society 2019.

8. Cox CE, Docherty SL, Brandon DH, Whaley C, Attix DK, Clay AS, Dore DV, Hough CL, White DB, Tulsky JA et al: Surviving critical illness: acute respiratory distress syndrome as experienced by patients and their caregivers. Critical care medicine 2009, 37(10):2702-2708. 
9. Haines KJ, Denehy L, Skinner EH, Warrillow S, Berney S: Psychosocial outcomes in informal caregivers of the critically ill: a systematic review. Critical care medicine 2015, 43(5):1112-1120.

10. Azoulay E, Pochard F, Kentish-Barnes N, Chevret S, Aboab J, Adrie C, Annane D, Bleichner G, Bollaert $\mathrm{PE}$, Darmon M: Risk of post-traumatic stress symptoms in family members of intensive care unit patients. American journal of respiratory and critical care medicine 2005, 171(9):987-994.

11. Cox CE, Hough CL, Carson SS, White DB, Kahn JM, Olsen MK, Jones DM, Somers TJ, Kelleher SA, Porter LS: Effects of a Telephone- and Web-based Coping Skills Training Program Compared with an Education Program for Survivors of Critical IIlness and Their Family Members. A Randomized Clinical Trial. American Journal of Respiratory \& Critical Care Medicine 2018, 197(1):66-78.

12. Haines KJ, Quasim T, McPeake J: Family and support networks following critical illness. Critical care clinics 2018, 34(4):609-623.

13. Choi J, Donahoe MP, Zullo TG, Hoffman LA: Caregivers of the chronically critically ill after discharge from the intensive care unit: six months' experience. American Journal of Critical Care 2011, 20(1):12-23.

14. Henderson, P. Quasim, T. Shaw, M. et al: Intensive Care Syndrome: Promoting Independence and Return to Employment. An evaluation of a multicentre critical care rehabilitation programme. THORAX (in press).

15. Von Elm, E. Altman, DG. Egger, M. et al (2007) Strengthening the reporting of observational studies in epidemiology (STROBE) statement: guidelines for reporting observational studies. BMJ;335.

16. McPeake JM, Henderson P, Darroch G, Iwashyna TJ, MacTavish P, Robinson C, Quasim T: Social and economic problems of ICU survivors identified by a structured social welfare consultation. Critical Care (London, England), 23(1):153.

17. McPeake J, Iwashyna TJ, Devine H, MacTavish P, Quasim TJT: Peer support to improve recovery following critical care discharge: a case-based discussion. Thorax 2017, 72(9):856-858.

18. Robinson C, Hibbert E, Bastin AJ, Meyer J, Montgomery-Yates A, Quasim T, Slack A, Mikkelsen ME, Iwashyna TJ, Haines KJ: An International Study Exploring the Experience of Survivors of Critical Illness as Volunteers Within ICU Recovery Services. Critical Care Explorations 2020, 2(11):e0273.

19. McPeake J, Boehm LM, Hibbert E, Bakhru RN, Bastin AJ, Butcher BW, Eaton TL, Harris W, Hope AA, Jackson J: Key components of ICU recovery programs: what did patients report provided benefit? Critical care explorations 2020, 2(4).

20. McPeake J, Shaw M, Iwashyna TJ, Daniel M, Devine H, Jarvie L, Kinsella J, MacTavish P, Quasim T: Intensive care syndrome: promoting independence and return to employment (InS: PIRE). Early evaluation of a complex intervention. PloS one 2017, 12(11):e0188028.

21. McPeake J, Devine H, MacTavish P, Fleming L, Crawford R, Struthers R, Kinsella J, Daniel M, Shaw M, Quasim T: Caregiver strain following critical care discharge: An exploratory evaluation. Journal of Critical Care 2016, 35:180-184.

22. Government S: Scottish index of multiple deprivation. In.: Scottish Government Edinburgh; 2016. 
23. Zigmond AS, Snaith RP: The hospital anxiety and depression scale. Acta psychiatrica scandinavica 1983, 67(6):361-370.

24. Robinson BC: Validation of a caregiver strain index. Journal of gerontology 1983, 38(3):344-348.

25. Bastien $\mathrm{CH}$, Vallières $\mathrm{A}$, Morin $\mathrm{CM}$ : Validation of the Insomnia Severity Index as an outcome measure for insomnia research. Sleep medicine 2001, 2(4):297-307.

26. Haines KJ, Beesley SJ, Hopkins RO, McPeake J, Quasim T, Ritchie K, Iwashyna TJ: Peer support in critical care: a systematic review. Critical care medicine 2018, 46(9):1522-1531.

27. Hope AA, Johnson A, McPeake J, Felt H, Sevin CM, Mikkelsen ME, Iwashyna TJ, Lassen-Greene C, Haines KJ, Agarwal S: Establishing a peer support program for survivors of COVID-19: a report from the Critical and Acute Illness Recovery Organization. American Journal of Critical Care 2021, 30(2):150-154.

28. Woodhead C, Khondoker M, Lomas R, Raine R: Impact of co-located welfare advice in healthcare settings: prospective quasi-experimental controlled study. The British Journal of Psychiatry 2017, 211(6):388-395.

29. McPeake JI, TJ. Henderson, P: Outcomes following critical care: A prospective cohort study of UK Biobank participants. Lancet Regional (Europe) (In press) 2021.

30. Rabiee A, Nikayin S, Hashem MD, Huang M, Dinglas VD, Bienvenu OJ, Turnbull AE, Needham DM: Depressive Symptoms After Critical Illness: A Systematic Review and Meta-Analysis. Critical care medicine 2016, 44(9):1744-1753.

31. Choi J, Sherwood PR, Schulz R, Ren D, Donahoe MP, Given B, Hoffman LA: Patterns of depressive symptoms in caregivers of mechanically ventilated critically ill adults from ICU admission to two months post-ICU discharge: A Pilot Study. Critical care medicine 2012, 40(5):1546.

32. Griffith DM, Salisbury LG, Lee RJ, Lone N, Merriweather JL, Walsh TS: Determinants of Health-Related Quality of Life After ICU: Importance of Patient Demographics, Previous Comorbidity, and Severity of Illness. Critical care medicine 2018, 46:N.PAG-N.PAG.

\section{Tables}

Table 1: Caregiver demographics, alongside paired patient demographics. 


\begin{tabular}{|c|c|c|c|}
\hline & Usual Care cohort & Intervention cohort & $p$ value \\
\hline & $(n=89)$ & $(n=81)$ & \\
\hline \multicolumn{4}{|l|}{ Caregiver demographics } \\
\hline Relationship with patient, n (\%) & & & 0.47 \\
\hline Partner or Spouse & $66(74.2)$ & $54(66.6)$ & \\
\hline Child or grandchild & $16(18)$ & $13(16.1)$ & \\
\hline Parent & $3(3.3)$ & $8(9.9)$ & \\
\hline Other & $4(4.5)$ & $6(7.4)$ & \\
\hline Age, years, median (IQR) & $58.0(47.7-69.0)$ & $58.0(48.0-66.3)$ & 0.88 \\
\hline Gender, male, n (\%) & $32(36.0)$ & $30(37.0)$ & 0.79 \\
\hline Socio-economic status & & & 0.50 \\
\hline SIMD 1 (most deprived) & $25(28.1)$ & $20(24.7)$ & \\
\hline SIMD 2 & $19(21.3)$ & $19(23.4)$ & \\
\hline SIMD 3 & $12(13.5)$ & $16(19.8)$ & \\
\hline SIMD 4 & $12(13.5)$ & $7(8.6)$ & \\
\hline SIMD 5 (least deprived) & $19(21.3)$ & $11(13.6)$ & \\
\hline \multicolumn{4}{|l|}{ Patient Demographics } \\
\hline Age at ICU admission, median (IQR) & $63.9(49.7-71.4)$ & $58.7(51.1-67.7)$ & 0.16 \\
\hline Gender, male, number (\%) & $50(56.2)$ & $41(50.6)$ & 0.33 \\
\hline ICU LOS, days, median (IQR) & $4.75(2.4-9.6)$ & $11.3(7.0-19.7)$ & $<0.01$ \\
\hline Hospital LOS, days, median (IQR) & $19.0(11.2-33.5)$ & $32.0(17.0-51.7)$ & $<0.01$ \\
\hline APACHE II, median (IQR) & $19.0(14.9-25.0)$ & $19.0(15.0-26.0)$ & 0.49 \\
\hline Mental Health issues pre-ICU, n (\%) & $20(22.5)$ & $21(25.9)$ & 0.72 \\
\hline Ventilation Required, n (\%) & $76(85.4)$ & $77(95.1)$ & 0.12 \\
\hline Two or more comorbidities, n (\%) & $42(47.2)$ & $34(42)$ & 0.34 \\
\hline Surgical admission & $42(47.2)$ & $31(38.3)$ & 0.17 \\
\hline $\begin{array}{l}\text { Time to follow up, } \\
\text { median months (IQR) }\end{array}$ & $15.0(13.1-16.5)$ & $16.0(14.8-17.5)$ & $<0.01$ \\
\hline
\end{tabular}


IQR: Interquartile Range; SIMD: Scottish Index of Multiple Deprivation; ICU: Intensive Care Unit; LOS: Length of Stay; APACHE II: Acute Physiology and Chronic Health Evaluation. Missing data information available in $\mathbf{S 2}$.

Table 2: Summary of the effect of InS:PIRE on study outcomes at 12 months estimated via Logistic Regression Models

\begin{tabular}{|llll|}
\hline Outcome measure & Adjusted estimate & P value & 95\% confidence interval \\
\hline HADS anxiety & 0.42 & $\mathbf{0 . 0 2}$ & $0.20-0.89$ \\
\hline HADS depression & 0.58 & 0.19 & $0.26-1.31$ \\
\hline Carer Strain & 0.39 & $\mathbf{0 . 0 4}$ & $0.16-0.98$ \\
\hline Clinical Insomnia & 0.36 & $<0.001$ & $0.17-0.77$ \\
\hline
\end{tabular}

\section{Figures}

\section{Figure 1}

Coefficients estimates demonstrating the impact of the InS:PIRE programme on caregiver outcomes at one year. Odds ratio of risk of screening positive for each outcome measure at one year in the InS:PIRE intervention cohort compared to the usual care cohort. Estimate with $95 \%$ confidence interval. Anxiety and depression: measured by Hospital Anxiety and Depression Scale (HADS), positive screening if score $\geq 8 / 21$ for each component score; Carer strain: measured by Carer Strain Index (CSI) with positive screening defined as a score of $\geq 7 / 13$; Insomnia: measured using the Insomnia Severity Index (ISI) with positive screening defined as a score of $\geq 8 / 28$.

\section{Supplementary Files}

This is a list of supplementary files associated with this preprint. Click to download.

- S1Caregiver.docx

- S2Caregivermissingvalues.docx

- S3caregiverpaperallregressionmodelsJan.docx 